\title{
Incoherent wind velocity sensing with a two-beam interferometer
}

\author{
Ralph R. Berggren \\ Los Alamos National Laboratory, E526, Los Alamos, NM 87545 \\ Phone 505-667-0593, fax 505-665-6834, xalphb@lanl.gov \\ David C. Thompson \\ Los Alamos National Laboratory, E548, Los Alamos, NM 87545 \\ Phone 505-667-5168, fax 505-665-4026, dcthomp@lanleov \\ R. J. Winkel, Jr. \\ Dept of Physics, U. S. Military Academy, West Point, NY 10996-1790 \\ Phone 845-938-3901, fax 845-938-5803,hr9304@usma.edu
}

\begin{abstract}
Analysis and laboratory demonstrations show meter per second accuracy with relatively loose tolerances and a small number of received photons. Gaussian error statistics lead to a simple and flexible approach to calculating wind fields.

(C2000 Optical Society of America

OCIS codes: (280.3340) Laser Doppler velocimetry; (120.3180) Interferometry
\end{abstract}

\section{Introduction}

Experience in optical testing has shown that fringe positions can be determined accurately with a two-beam interferometer and that very long optical path differences can be used [1]. Whether in the form of a Fizeau or an unequal-path Twyman-Green interferometer testing uncoated optics, fringe position accuracies and wavefront error are regularly obtained with a standard deviation of $1 / 100$ wave or better. The fringe-finding accuracy and the operation with an unequal path can be combined to measure very small wavelength shifts. A sensitive measurement of velocity can be made by using a narrow-line laser source combined with a receiver containing a modified MachZehnder interferometer having one arm substantially longer than the other. Although the use of two-beam interferometry is common in optical testing, it has received little discussion in regard to wind sensing. However, at least one device based on a Michelson interferometer [2] is known to exist.

The sensitivity of the system is a direct function of the optical path difference (OPD) between the arms. A measurement is made by introducing one or more fringes of tilt into the interferometer and observing the resulting fringe shift. The accuracy with which the fringe shift can be measured depends on the number of photons received, giving a normally distributed (Gaussian) distribution of measured errors. This leads to a simple and flexible approach for measuring two-dimensional or three-dimensional wind fields.

\section{Basic equations}

A wavefront incident on the interferometer reaches the detector as two waves having a phase difference, $\phi$, in waves, that depends on the OPD and the wavenumber $v$, giving $\phi=(O P D) v$. Laser light scattered back from the region of interest has its wavenumber shifted by an amount $d v=2 \mathrm{vV} / \mathrm{c}$, where $\mathrm{V}$ is the wind velocity in the direction of the line of sight and $c$ is the velocity of light. Combing these two equations gives a phase shift $d \phi=2$ (OPD) $v \mathrm{~V} / \mathrm{c}$. Writing this in terms of the wavelength as $\mathrm{d} \phi=(\mathrm{OPD} / \lambda)(2 \mathrm{~V} / \mathrm{c})$ gives a more intuitive form. The first term, $\mathrm{OPD} / \lambda$, is very large; the second, $2 \mathrm{~V} / \mathrm{c}$, is very small. The result is an easily observable $d \phi$. As an example, for a wavelength of $350 \mathrm{~nm}$ and $O P D=1$ meter, the phase shift for $V=1 \mathrm{~m} / \mathrm{s}$ is $1 / 50$ wave.

\section{Optical layout}

A representative layout is shown below. It is assumed that the interferometer is fed by an afocal telescope having an exit pupil at or near the final optical element. This might be a Cassegrain or a three-mirror anastigmat configuration, with a field lens at its image producing a pupil at a following collimator optic. The pupil is then relayed through each arm of the interferometer with a two-lens Fourier transform relay. Interference takes place on a detector placed at the relayed pupil. The separations between the pupils and lenses, and between each lens and the intermediate image, are equal to the focal lengths of the lenses. This is a $4 f$ (4-focal-length) relay. The focal 


\section{DISCLAIMER}

This report was prepared as an account of work sponsored by an agency of the United States Government. Neither the United States Government nor any agency thereof, nor any of their employees, make any warranty, express or implied, or assumes any legal liability or responsibility for the accuracy, completeness, or usefulness of any information, apparatus, product, or process disclosed, or represents that its use would not infringe privately owned rights. Reference herein to any specific commercial product, process, or service by trade name, trademark, manufacturer, or otherwise does not necessarily constitute or imply its endorsement, recommendation, or favoring by the United States Government or any agency thereof. The views and opinions of authors expressed herein do not necessarily state or reflect those of the United States Government or any agency thereof. 


\section{DISCLAIMER}

Portions of this document may be illegible in electronic image products. Images are produced from the best available original document. 
lengths of the first and second lenses may be different in order to adjust the pupil size to the desired detector size, as long as the proper separations are maintained. Using the parameters given above, the two arms might have lengths of 0.5 and 1.5 meters, with the lenses having focal lengths of 125 and $375 \mathrm{~mm}$. The important aspects of the $4 \mathrm{f}$ relay are that the wavefront is reproduced exactly and the optical path length is identical for all field points. The system

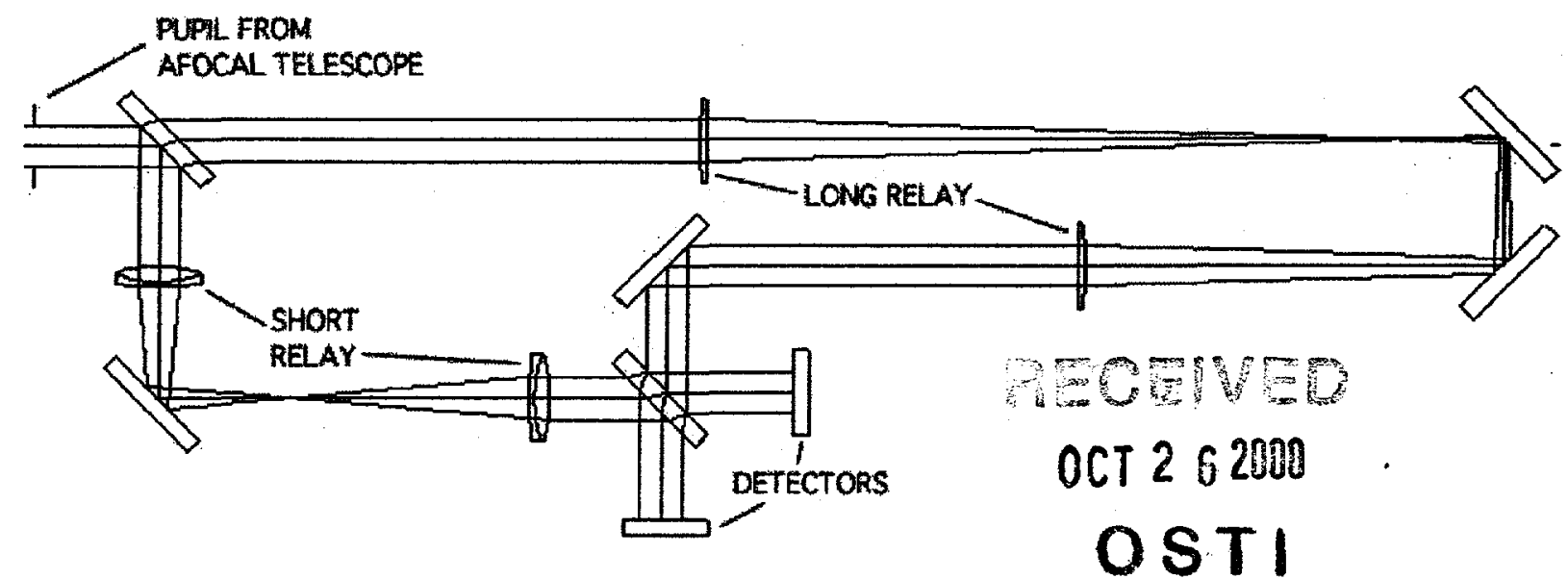

works over as wide a field as desired, limited only by the aberrations of the lenses. As a result, a large input field and a small-aperture interferometer can be used. For a $1-\mathrm{m}$ diameter telescope and a $25-\mathrm{mm}$ pupil in the interferometer, a 1-mrad field of view would require only a 40-mrad field angle in the interferometer, a relatively easy design problem. The interferometer has two outputs, allowing the use of two detectors, and eliminating reflections back out of the system. The detectors should be imaging photomultipliers, allowing the fringe position to be determined with few detected photons.

\section{Mode of operation and performance prediction}

It is assumed that a laser source with a sufficiently narrow spectral line is used in a pulsed mode so that range gating can be used to separate the returns from different regions of the atmosphere. The scattered returns will be collected by the afocal telescope. The interferometer is adjusted to give one or more fringes on the detectors, with two detectors used as shown. Speckle from the scattered return will result in non-uniform illumination of the aperture at any moment. In normal operation, a wind sensor would not be used with high spatial resolution, so the transmitted beam could cover many times the diffraction limit, giving a number of speckles across the aperture. A small amount of the output laser beam would be directed into the interferometer as a reference, using the second input available in the Mach-Zehnder configuration, to allow the fringe position and spacing to be determined, and to permit realignment of the interferometer if needed.

A simple computer model was created to predict the performance of such a system. In the model, a photon is assumed to be incident with a given shifted wavelength at a random position in the aperture. With appropriate probabilities depending on the photon position in the fringe pattern, the photon reaches one of the detectors and is detected with an assumed quantum efficiency.

After a given number of photons has been counted the phase is calculated. Each imaging photomultiplier will have a number of photon counts for each detector element. Since the fringe period is known, a linear least-squares fit can be used to determine the coefficients of sine and cosine terms, and thus to determine the phase.

Analysis showed that, as expected[3], the rms error in determining the phase is inversely proportional to the square root of the number of photon counts, and that an rms error of $1 / 50$ wave is obtained with fewer than 100 counts. For the example given above, with a 1-meter OPD, an rms velocity error of $1 \mathrm{~m} / \mathrm{s}$ can be expected from 100 counts. An important result is that it makes no difference whether this is done with one measurement of 100 counts or with 10 measurements of 10 counts. This is an important property that has a significant impact on data analysis.

\section{Aerosol and molecular scattering}

For measurement of scatter from aerosols there is no significant Doppler broadening of the laser line and a large OPD can be used. For molecular scattering the Doppler broadening is important. The high molecular velocities give rise to a broadened line with a half-width of about $180 \mathrm{~m} / \mathrm{s}$. The OPD must be small enough so that this broadening creates less than one fringe phase change, which requires that the fringes must be located more accurately for a given velocity accuracy. For pure molecular scattering, the computer model indicated an optimum OPD of about 50 
$\mathrm{mm}$ and a need for somewhat more than 10,000 photon counts. For mixed aerosol and molecular scattering when the aerosol is a few percent or more of the total, it is best to use the long OPD. The molecular scattering then acts as a uniform background and increases the number of counts required beyond the 100 needed for pure aerosol measurements. Thus a two-interferometer system would usually be used, with the energy split between the two depending on particular system requirements.

\section{Experimental verification}

A breadboard system was created with a CW He-Ne laser focused on the rim of a spinning disk. The scattered light was directed through an interferometer as in the figure, with a 1.6-meter OPD, using a 5-mm diameter aperture. Fringe shifts were measured manually. Disk speed was measured with a tachometer. Measurements over a range of 0 to $10 \mathrm{~m} / \mathrm{s}$ (the maximum disk speed) agreed within $1 \mathrm{~m} / \mathrm{s}$.

In a separate measurement, an imaging photomultiplier was used in an interferometer to determine the accuracy of finding fringe position as a function of the number of photon counts. In this direct measurement, an rms error of 1/50 wave was obtained with fewer than 100 counts. The results confirmed the predicted performance both for good modulation, corresponding to pure aerosol scattering, and for a large background, corresponding to mixed aerosol and molecular scattering. As expected, down to the experimental accuracy limit of 1/100 wave rms, the measurement accuracy varied inversely with the number of photon counts.

\section{Calculating the 3-dimensional wind field}

The wind velocity field can be described by any desired set of functions of $x, y, z$. The simplest example would be to take a given small area and height and assume that the three velocity components are constant over that volume. Another approach would be to take a larger area and assume that the velocity varies linearly over the region. Or, the wind field might be fit to a higher-order function.

Each measurement gives the dot product of the wind velocity and the viewing direction. The measurements have to be taken such that there is a reasonable sampling of directions over the desired area. Solving for the three velocity components in the area of interest is a linear problem, permitting a standard linear least-squares solution.

An advantage of the least-squares solution is that it also shows how well the calculated results fit the input data. If the fit error matches that expected from the known number of photon counts, then it is known that the solution is as good as can be obtained. If the fit error is significantly larger than expected, then it is known that there is turbulence or other velocity variation over the sampled volume, and an estimate of the variation can be made.

A computer simulation showed that: 1) As expected, the accuracy of the results depends on the total number of photon counts, whether there are many poor measurements with few photons, or a few measurements with more photons. 2) It can be preferable to describe the wind in a functional form over a larger area than to characterize it as a set of constant terms over smaller areas. 3) Since the vertical velocity is expected to be small in most cases, it may be desirable to assume a zero value and solve for only the horizontal components.

It is concluded that an interferometer of this type offers good accuracy of velocity measurement with simple, small optics. It is easily calibrated, and provides an output that is well matched to a variety of data-analysis needs.

\section{References}

1. Daniel Malacara, Optical Shop Testing, John Wiley and Sons, New York (1970) Chapter 2.4

2. John E. Bertie, Imaging atmospheric winds and temperatures with optical Doppler Michelson interferometry: high-resolution spectroscopy at small optical retardations in Proc. SPIE Vol. 2089, 92098 (1994)

3. John Lawall and Ernest Kessler, Michelson interferometry with 10 pm accuracy, Rev. Sci. Instrum. 71, 2669-2676 (2000) 